\title{
PERIODIC ORBITS AND NON-INTEGRABILITY OF ARMBRUSTER-GUCKENHEIMER-KIM POTENTIAL
}

\author{
JAUME LLIBRE ${ }^{1}$ AND LUCI ROBERTO ${ }^{2}$
}

\begin{abstract}
In this paper we study the periodic orbits of the Hamiltonian system with the Armburster-Guckenheimer-Kim potential and its non-integrability in the sense of LiouvilleArnold.
\end{abstract}

\section{IntRoduCtion AND STATEMENT OF THE MAIN RESUlts}

The main goal of this work is to study the periodic orbits and the non-integrability of the Hamiltonian system with the potential energy given by the Armburster-Guckenheimer-Kim potential, (see [2]), which has often been used in the study of the dynamics of galaxies. We investigate the periodic orbits using the averaging theory and the non-integrability is studied through the existence of periodic orbits that do not have all their multipliers equal to 1.

This Hamiltonian consists of a two dimensional harmonic potential plus the following quartic terms

$$
\frac{1}{2}\left(p_{x}^{2}+p_{y}^{2}+x^{2}+y^{2}\right)-\frac{a}{4}\left(x^{2}+y^{2}\right)^{2}-\frac{b}{2} x^{2} y^{2} .
$$

The Hamiltonian system is given by

$$
\begin{aligned}
& \dot{x}=p_{x}, \\
& \dot{p}_{x}=-x+a x\left(x^{2}+y^{2}\right)+b x y^{2}, \\
& \dot{y}=p_{y}, \\
& \dot{p}_{y}=-y+a y\left(x^{2}+y^{2}\right)+b x^{2} y .
\end{aligned}
$$

As usual the dot denotes derivative with respect to the independent variable $t$, the time. We name (2) the Armburster-Guckenheimer-Kim Hamiltonian systems, or simply the AGK systems.

In this work we use the averaging method of first order to compute periodic orbits, see appendix 4.2. This method allows to find analytically periodic orbits of the AGK systems (2) at any positive values of the energy as a function of the parameters $a$ and $b$. Roughly speaking this method reduces the problem of finding periodic solutions of some differential system to the one of finding zeros of some convenient finite dimensional function. This method was also used by Llibre and Jiménez-Lara in $[4,5]$.

Our main result on the periodic orbits of the AGK system (2) is summarized as follows.

Theorem 1. At every positive energy level the Armbruster-Guckenheimer-Kim system (2) has at least:

(a) two periodic orbits if either $b=2 a \neq 0$ or $b=0$ and $a \neq 0$.

(b) or six periodic orbits if $b(a+b)(2 a-b) \neq 0$. 
Theorem 1 states that at any positive energy level there exist periodic orbits and we can use these particular periodic orbits to prove our second main result about the non-integrability in the sense of Liouville-Arnold of the AGK system (2).

Theorem 2. Assume that the AGK Hamiltonian system (2) satisfies the assumptions of one the statements of Theorem 1, and denote by (*) this statement. Then under the assumption of statement $(*)$

(a) either the AGK Hamiltonian system is Liouville-Arnold integrable and the gradients of the two constants of motion are linearly dependent on some points of the periodic orbits found in statement (*) of Theorem 1,

(b) or the AGK Hamiltonian system is not Liouville-Arnold integrable with any second first integral of class $\mathcal{C}^{1}$.

The proof of Theorem 1 is based on the averaging theory for computing periodic orbits, see the appendix 4.2. And the proof of Theorem 2 is based on the Poincaré's Method that allows to prove the non Liouville-Arnold integrability independently of the class of differentiability of the second first integral, see appendix 4.1 for more details. The main difficulty for applying Poincaré's non-integrability method to a given Hamiltonian system is to find for such system periodic orbits having multipliers different from 1. For applying the Poincaré non-integrability theory to AGK-system, we need to study some of the periodic orbits of these system and to computer their multipliers.

\section{Proof of TheOREM 1}

To prove Theorem 1 we shall apply Theorem 5 to the Hamilonian system (2). The periodic orbits of a Hamiltonian system of more than one degree of freedom are generically on cylinders fulfilled of periodic orbits in the phase space (see [1]), then we will not be able to apply directly Theorem 5 to a Hamiltonian system because then the corresponding averaged function $f_{1}$ at the equilibrium point $a$ will be always zero. This problem will be solved by fixing an energy level where the periodic orbits can be isolated.

To apply Theorem 5 we need a small parameter $\varepsilon$. In system (2) we consider the change of variables $\left(x, p_{x}, y, p_{y}\right) \mapsto\left(X, p_{X}, Y, p_{Y}\right)=\left(x / \sqrt{\varepsilon}, p_{x} / \sqrt{\varepsilon}, y / \sqrt{\varepsilon}, p_{y} / \sqrt{\varepsilon}\right)$. In the new variables, system (2) becomes

$$
\begin{aligned}
& \dot{X}=p_{X}, \\
& \dot{p}_{X}=-X+\varepsilon\left(a X^{3}+(a+b) X Y^{2}\right), \\
& \dot{Y}=p_{Y}, \\
& \dot{p}_{Y}=-Y+\varepsilon\left((a+b) X^{2} Y+a Y^{3}\right) .
\end{aligned}
$$

This system again is Hamiltonian with the Hamiltonian

$$
\frac{1}{2}\left(P^{2}+Q^{2}+X^{2}+Y^{2}\right)-\varepsilon \frac{a}{4}\left(X^{2}+Y^{2}\right)^{2}-\varepsilon^{2} \frac{b}{2} X^{2} Y^{2} .
$$

As the change of variables is only a scale transformation, for all $\varepsilon$ different from zero, the original and the transformed systems (2) and (3) have essentially the same phase portrait, and additionally system (3) for $\varepsilon$ sufficiently small is close to an integrable one.

Notice that system (3) is not in the normal form for applying the averaging theory, see the differential equation (18). We consider the change of variables

$$
X=r \cos \theta, \quad p_{X}=r \sin \theta, \quad Y=\rho \cos (\theta+\alpha), \quad p_{Y}=\rho \sin (\theta+\alpha) .
$$


Recall that this is a change of variables when $r>0$ and $\rho>0$. Moreover doing this change of variables appear in the system the periodic variables $\theta$ and $\alpha$. Later on the variable $\theta$ will be used for obtaining the periodicity necessary for applying the averaging theory.

The energy or Hamiltonian in these news variables becomes

$$
H=\frac{1}{2}\left(r^{2}+\rho^{2}\right)-\frac{1}{4} \varepsilon a\left(r^{2} \cos ^{2} \theta+\rho^{2} \cos ^{2}(\theta+\alpha)\right)^{2}-\frac{1}{2} \varepsilon^{2} b r^{2} \rho^{2} \cos ^{2} \theta \cos ^{2}(\theta+\alpha)
$$

and the equations of motion are given by

$$
\begin{aligned}
\dot{r} & =\varepsilon r \sin \theta \cos \theta\left(a r^{2} \cos ^{2} \theta+(a+b) \rho^{2} \cos ^{2}(\theta+\alpha)\right), \\
\dot{\theta} & =-1+\varepsilon\left(a r^{2} \cos ^{4} \theta+(a+b) \rho^{2} \cos ^{2} \theta \cos ^{2}(\theta+\alpha)\right), \\
\dot{\rho} & =\varepsilon \rho \sin (\theta+\alpha) \cos (\theta+\alpha)\left((a+b) r^{2} \cos ^{2} \theta+a \rho^{2} \cos ^{2}(\theta+\alpha)\right), \\
\dot{\alpha} & =\varepsilon\left(-a r^{2} \cos ^{4} \theta+(a+b)\left(r^{2}-\rho^{2}\right) \cos ^{2} \theta \cos ^{2}(\theta+\alpha)+a \rho^{2} \cos ^{4}(\theta+\alpha)\right) .
\end{aligned}
$$

However the derivatives of the left hand side of these equations are with respect to the time variable $t$, which is not periodic. We change to the $\theta$ variable as the independent one, and we denote by a prime the derivative with respect to $\theta$. Then system (6) goes over to

$$
\begin{aligned}
r^{\prime} & =\frac{\varepsilon r \sin \theta \cos \theta\left(a r^{2} \cos ^{2} \theta+(a+b) \rho^{2} \cos ^{2}(\theta+\alpha)\right)}{-1+a r^{2} \varepsilon \cos ^{4} \theta+(a+b) \varepsilon \rho^{2} \cos ^{2} \theta \cos ^{2}(\theta+\alpha)}, \\
\rho^{\prime} & =\frac{\varepsilon \rho \sin (\theta+\alpha) \cos (\theta+\alpha)\left((a+b) r^{2} \cos ^{2} \theta+a \rho^{2} \cos ^{2}(\theta+\alpha)\right)}{-1+a r^{2} \varepsilon \cos ^{4} \theta+(a+b) \varepsilon \rho^{2} \cos ^{2} \theta \cos ^{2}(\theta+\alpha)}, \\
\alpha^{\prime} & =\frac{\varepsilon\left(-a r^{2} \cos ^{4} \theta+(a+b)\left(r^{2}-\rho^{2}\right) \cos ^{2} \theta \cos ^{2}(\theta+\alpha)+a \rho^{2} \cos ^{4}(\theta+\alpha)\right)}{-1+a r^{2} \varepsilon \cos ^{4} \theta+(a+b) \varepsilon \rho^{2} \cos ^{2} \theta \cos ^{2}(\theta+\alpha)} .
\end{aligned}
$$

Of course this system has now only three equations because we do not need the $\theta$ equation. If we write the previous system as a Taylor series in powers of $\varepsilon$, we have

$$
\begin{aligned}
& r^{\prime}=-\varepsilon r \sin \theta \cos \theta\left(a r^{2} \cos ^{2} \theta+(a+b) \rho^{2} \cos ^{2}(\theta+\alpha)\right)+O\left(\varepsilon^{2}\right), \\
& \rho^{\prime}=-\varepsilon \rho \sin (\theta+\alpha) \cos (\theta+\alpha)\left((a+b) r^{2} \cos ^{2} \theta+a \rho^{2} \cos ^{2}(\theta+\alpha)\right)+O\left(\varepsilon^{2}\right), \\
& \alpha^{\prime}=\varepsilon\left(a r^{2} \cos ^{4} \theta-(a+b)\left(r^{2}-\rho^{2}\right) \cos ^{2} \theta \cos ^{2}(\theta+\alpha)-a \rho^{2} \cos ^{4}(\theta+\alpha)\right)+O\left(\varepsilon^{2}\right) .
\end{aligned}
$$

Now system ( 7 ) is $2 \pi$-periodic in the variable $\theta$. We shall apply Theorem 5 in the Hamiltonian level $H=h$ for $h>0$, and by solving equation $H=h$ for $\rho$ we obtain

$$
\rho=\sqrt{-\frac{\sec ^{4}(\alpha+\theta) A+\varepsilon r^{2} \cos ^{2} \theta(a+b \varepsilon) \sec ^{2}(\alpha+\theta)}{a \varepsilon},}
$$

where

$$
A=-1+\sqrt{1+\varepsilon \cos ^{4}(\alpha+\theta)\left(\varepsilon^{2} b(2 a+b \varepsilon) r^{4} \cos ^{4} \theta+2 a\left(r^{2}-2 h\right)\right)-2 \varepsilon r^{2} \cos ^{2} \theta(a+b \varepsilon) \cos ^{2}(\alpha+\theta)} .
$$

Then substituting $\rho$ in equations (7) and developing in power series of $\varepsilon$, we obtain the two differential equations

$$
\begin{aligned}
r^{\prime}= & \varepsilon r \sin \theta \cos \theta\left((a+b)\left(r^{2}-2 h\right) \cos ^{2}(\alpha+\theta)-a r^{2} \cos ^{2} \theta\right)+O\left(\varepsilon^{2}\right), \\
\alpha^{\prime}= & \varepsilon\left(2(a+b)\left(h-r^{2}\right) \cos ^{2} \theta \cos ^{2}(\alpha+\theta)+\right. \\
& \left.a\left(r^{2}-2 h\right) \cos ^{4}(\alpha+\theta)+a r^{2} \cos ^{4} \theta\right)+O\left(\varepsilon^{2}\right) .
\end{aligned}
$$

Clearly system (9) satisfies the assumptions of Theorem 5 and it has the form (18) with $F_{1}=\left(F_{11}, F_{12}\right)$, given by

$$
\begin{aligned}
& F_{11}=r \sin \theta \cos \theta\left((a+b)\left(r^{2}-2 h\right) \cos ^{2}(\alpha+\theta)-a r^{2} \cos ^{2} \theta\right), \\
& F_{12}=2(a+b)\left(h-r^{2}\right) \cos ^{2} \theta \cos ^{2}(\alpha+\theta)+a\left(r^{2}-2 h\right) \cos ^{4}(\alpha+\theta)+a r^{2} \cos ^{4} \theta .
\end{aligned}
$$


Notice that $F_{1}$ is $2 \pi$-periodic in the variable $\theta$, the independent variable of system (9). Averaging the function $F_{1}$ with respect to the variable $\theta$ we have

$$
f_{1}(r, \alpha)=\left(f_{11}(r, \alpha), f_{12}(r, \alpha)\right)=\frac{1}{2 \pi} \int_{0}^{2 \pi}\left(F_{11}, F_{12}\right) d \theta
$$

where

$$
f_{11}(r, \alpha)=-\frac{1}{8} r(a+b)\left(r^{2}-2 h\right) \sin (2 \alpha)
$$

and

$$
f_{12}(r, \alpha)=\frac{1}{4}\left(h-r^{2}\right)((a+b) \cos (2 \alpha)-a+2 b) .
$$

We must find the zeros $\left(r^{*}, \alpha^{*}\right)$ of $f_{1}(r, \alpha)$, and check that the Jacobian at these points is not zero, i.e.

$$
\operatorname{det}\left(\left.\frac{\partial\left(f_{11}, f_{12}\right)}{\partial(r, \alpha)}\right|_{(r, \alpha)=\left(r^{*}, \alpha^{*}\right)}\right) \neq 0 .
$$

From $f_{11}(r, \alpha)=0$ we obtain that either $r=0$ or, $r= \pm \sqrt{2 h}$ or $\alpha=0, \pi / 2,-\pi / 2, \pi$. The solutions $r=0$ and $r=-\sqrt{2 h}$ are not good, because $r>0$. So, the good solutions of $f_{11}(r, \alpha)=0$ are $r=\sqrt{2 h}$ and $\alpha=0, \pi / 2,-\pi / 2, \pi$. Now we look for the solutions of $f_{12}(r, \alpha)=0$. We obtain nine possible solutions $\left(r^{*}, \alpha^{*}\right)$ with $r^{*}>0$ :

$$
\begin{aligned}
& s_{1}=(\sqrt{h}, 0), s_{2}=(\sqrt{h}, \pi), s_{3}=(\sqrt{h}, \pi / 2), s_{4}=(\sqrt{h}, 3 \pi / 2), s_{5}=(\sqrt{2 h}, 0), s_{6}=(\sqrt{2 h}, \pi) \\
& s_{7}=(\sqrt{2 h}, \pi / 2), s_{8}=\left(\sqrt{2 h}, \frac{1}{2} \arccos \left(\frac{a-2 b}{a+b}\right)\right), s_{9}=\left(\sqrt{2 h}, 2 \pi-\frac{1}{2} \arccos \left(\frac{a-2 b}{a+b}\right)\right),
\end{aligned}
$$

with corresponding values of $\rho$ given by (8) tending to $\sqrt{h}$ for the solutions $s_{1}, s_{2}, s_{3}, s_{4}$ when $\varepsilon \rightarrow 0$ and tending to 0 for the solutions $s_{5}, s_{6}, s_{7}, s_{8}, s_{9}$ when $\varepsilon \rightarrow 0$. Of course in (11) for the solutions $s_{8}$ and $s_{9}$ we assume that $-1 \leq(a-2 b) /(a+b) \leq 1$.

Finally we calculate the Jacobian (10), i.e.

$$
\left|\begin{array}{cc}
\frac{1}{4}(a+b) \pi\left(2 h-3 r^{2}\right) \sin (2 \alpha) & -\frac{1}{2}(a+b) \pi r\left(r^{2}-2 h\right) \cos (2 \alpha) \\
-\pi r(-a+2 b+(a+b) \cos (2 \alpha)) & (-a-b) \pi\left(h-r^{2}\right) \sin (2 \alpha)
\end{array}\right|
$$

at the nine solutions $s_{1}, \ldots, s_{9}$. Then we obtain the Jacobian

$$
\frac{3}{8} h^{2} b(a+b)
$$

at the solutions $s_{1}$ and $s_{2}$, the Jacobian

$$
\frac{1}{8} h^{2}(2 a-b)(a+b)
$$

at the solutions $s_{3}$ and $s_{4}$, the Jacobian 0 at the solutions $s_{5}, s_{6}$ and $s_{7}$, and the Jacobian

$$
\frac{3}{4} h^{2} b(b-2 a)
$$

at the solutions $s_{8}$ and $s_{9}$.

Notice that the above Jacobian at the solutions $s_{5}, s_{6}$ and $s_{7}$ are zero, then we cannot use Theorem 5 for these solutions. However we have that for $h \neq 0$ the Jacobian is non-zero at $s_{1}$ and $s_{2}$ when

$$
b(a+b) \neq 0,
$$


the Jacobian is non-zero at $s_{3}$ and $s_{4}$ when

$$
(2 a-b)(a+b) \neq 0
$$

and the Jacobian is non-zero at $s_{8}$ and $s_{9}$ when

$$
b(b-2 a) \neq 0 .
$$

Summarizing, from Theorem 5 , the solutions $s_{1}$ and $s_{2}$ of $f\left(r^{*}, \alpha^{*}\right)=0$ provide two periodic solutions of system (9) (and consequently of the Hamiltonian system (3) on the level $h>0$ ) if $b \neq 0$ and $b=2 a$. Similarly, the solutions $s_{3}$ and $s_{4}$ provide two periodic solutions of system (9) if $a \neq 0$ and $b=0$. And, if $b(a+b)(2 a-b) \neq 0$ the solutions $s_{i}$ for $i=1,2,3,4,8,9$ of $f_{1}=0$ provide at least six periodic solutions for the Hamiltonian system (3). This completes the proof of Theorem 1.

Note that if $a+b=0$, then we do not have any periodic solution given by $s_{i}$ for $i=1,2,3,4,8,9$ because either their Jacobian is zero (for $i=1,2,3,4$ ) or they are not defined (for $i=8,9$, see $(11))$.

\section{Proof of Theorem 2}

We assume that we are under the assumptions of Theorem 1, and that one of the six founded periodic solutions corresponding to the solutions $s_{1}, s_{2}, s_{3}, s_{4}, s_{8}$ and $s_{9}$ exist, and that their associated Jacobians (13), (14) and (15) are non-zero. So the correponding multipliers are not all equal to 1 . Hence under the assumptions of Theorem 1, by Theorem 4, either the AGKsystems cannot be Liouville-Arnold integrable with any second first integral $G$ or these systems are Liouville-Arnold integrable and the vector gradient of $H$ and $G$ are linearly dependent on some points of these periodic orbits. Therefore Theorem 2 is proved.

\section{Appendix}

4.1. Periodic orbits and the Liouville-Arnold integrability. We shall summarize some facts on the Liouville-Arnold integrability of the Hamiltonian systems, and on the theory of the periodic orbits of the differential equations, for more details see $[1,3]$ and the subsection 7.1.2 of [3], respectively. We present these results for Hamiltonian systems of two degrees of freedom, because we are studying a Hamiltonian system with two degrees of freedom associated to the motion of generalized Henon-Heiles systems, but these results work for an arbitrary number of degrees of freedom.

We recall that a Hamiltonian system with Hamiltonian $H$ of two degrees of freedom is integrable in the sense of Liouville-Arnold if it has a first integral $G$ independent with $H$ (i.e. the gradient vectors of $H$ and $G$ are independent in all the points of the phase space except perhaps in a set of zero Lebesgue measure), and in involution with $H$ (i.e. the parenthesis of Poisson of $H$ and $G$ is zero). For Hamiltonian systems with two degrees of freedom the involution condition is redundant, because the fact that $G$ is a first integral of the Hamiltonian system, implies that the mentioned Poisson parenthesis is always zero. A flow defined on a subspace of the phase space is complete if its solutions are defined for all time.

Now we shall state the Liouville-Arnold Theorem restricted to Hamiltonian systems of two degrees of freedom.

Theorem 3. Suppose that a Hamiltonian system with two degrees of freedom defined on the phase space $M$ has its Hamiltonian $H$ and the function $G$ as two independent first integrals in involution. If $I_{h c}=\{p \in M: H(p)=h$ and $C(p)=c\} \neq \emptyset$ and $(h, c)$ is a regular value of the map $(H, G)$, then the following statements hold. 
(a) $I_{h c}$ is a two dimensional submanifold of $M$ invariant under the flow of the Hamiltonian system.

(b) If the flow on a connected component $I_{h c}^{*}$ of $I_{h c}$ is complete, then $I_{h c}^{*}$ is diffeomorphic either to the torus $\mathbb{S}^{1} \times \mathbb{S}^{1}$, or to the cylinder $\mathbb{S}^{1} \times \mathbb{R}$, or to the plane $\mathbb{R}^{2}$. If $I_{h c}^{*}$ is compact, then the flow on it is always complete and $I_{h c}^{*} \approx \mathbb{S}^{1} \times \mathbb{S}^{1}$.

(c) Under the hypothesis (b) the flow on $I_{h c}^{*}$ is conjugated to a linear flow on $\mathbb{S}^{1} \times \mathbb{S}^{1}$, on $\mathbb{S}^{1} \times \mathbb{R}$, or on $\mathbb{R}^{2}$.

The main result of this theorem is that the connected components of the invariant sets associated with the two independent first integrals in involution are generically submanifolds of the phase space, and if the flow on them is complete then they are diffeomorphic to a torus, a cylinder or a plane, where the flow is conjugated to a linear one.

Using the notation of Theorem 3 when a connected component $I_{h c}^{*}$ is diffeomorphic to a torus, either all orbits on this torus are periodic if the rotation number associated to this torus is rational, or they are quasi-periodic (i.e. every orbit is dense in the torus) if the rotation number associated to this torus is not rational.

We consider the autonomous differential system

$$
\dot{x}=f(x),
$$

where $f: U \rightarrow \mathbb{R}^{n}$ is $C^{2}, U$ is an open subset of $\mathbb{R}^{n}$ and the dot denotes the derivative respect to the time $t$. We write its general solution as $\phi\left(t, x_{0}\right)$ with $\phi\left(0, x_{0}\right)=x_{0} \in U$ and $t$ belonging to its maximal interval of definition.

We say that $\phi\left(t, x_{0}\right)$ is $T$-periodic with $T>0$ if and only if $\phi\left(T, x_{0}\right)=x_{0}$ and $\phi\left(t, x_{0}\right) \neq x_{0}$ for $t \in(0, T)$. The periodic orbit associated to the periodic solution $\phi\left(t, x_{0}\right)$ is $\gamma=\left\{\phi\left(t, x_{0}\right), t \in\right.$ $[0, T]\}$. The variational equation associated to the $T$-periodic solution $\phi\left(t, x_{0}\right)$ is

$$
\dot{M}=\left(\left.\frac{\partial f(x)}{\partial x}\right|_{x=\phi\left(t, x_{0}\right)}\right) M,
$$

where $M$ is an $n \times n$ matrix. The monodromy matrix associated to the $T$-periodic solution $\phi\left(t, x_{0}\right)$ is the solution $M\left(T, x_{0}\right)$ of $(17)$ satisfying that $M\left(0, x_{0}\right)$ is the identity matrix. The eigenvalues $\lambda$ of the monodromy matrix associated to the periodic solution $\phi\left(t, x_{0}\right)$ are called the multipliers of the periodic orbit.

For an autonomous differential system, one of the multipliers is always 1, and its corresponding eigenvector is tangent to the periodic orbit.

A periodic solution of an autonomous Hamiltonian system always has two multipliers equal to one. One multiplier is 1 because the Hamiltonian system is autonomous, and another is 1 due to the existence of the first integral given by the Hamiltonian.

Theorem 4. If a Hamiltonian system with two degrees of freedom and Hamiltonian $H$ is Liouville-Arnold integrable, and $G$ is a second first integral such that the gradients of $H$ and $G$ are linearly independent at each point of a periodic orbit of the system, then all the multipliers of this periodic orbit are equal to 1.

Theorem 4 is due to Poincaré [6]. It gives us a tool to study the non Liouville-Arnold integrability, independently of the class of differentiability of the second first integral. The main problem for applying this theorem is to find periodic orbits having multipliers different from 1 . 
4.2. Averaging Theory of First Order. Now we shall present the basic results from averaging theory that we need for proving the results of this paper.

The next theorem provides a first order approximation for the periodic solutions of a periodic differential system, for the proof see Theorems 11.5 and 11.6 of Verhulst [7].

Consider the differential equation

$$
\dot{\mathbf{x}}=\varepsilon F_{1}(t, \mathbf{x})+\varepsilon^{2} F_{2}(t, \mathbf{x}, \varepsilon), \quad \mathbf{x}(0)=\mathbf{x}_{0}
$$

with $\mathbf{x} \in D \subset \mathbb{R}^{n}, t \geq 0$. Moreover we assume that both $F_{1}(t, \mathbf{x})$ and $F_{2}(t, \mathbf{x}, \varepsilon)$ are $T$-periodic in $t$. Separately we consider in $D$ the averaged differential equation

$$
\dot{\mathbf{y}}=\varepsilon f_{1}(\mathbf{y}), \quad \mathbf{y}(0)=\mathbf{x}_{0},
$$

where

$$
f_{1}(\mathbf{y})=\frac{1}{T} \int_{0}^{T} F_{1}(t, \mathbf{y}) d t .
$$

Under certain conditions, equilibrium solutions of the averaged equation turn out to correspond with $T$-periodic solutions of equation (18).

Theorem 5. Consider the two initial value problems (18) and (19). Suppose:

(i) $F_{1}$, its Jacobian $\partial F_{1} / \partial x$, its Hessian $\partial^{2} F_{1} / \partial x^{2}, F_{2}$ and its Jacobian $\partial F_{2} / \partial x$ are defined, continuous and bounded by a constant independent of $\varepsilon$ in $[0, \infty) \times D$ and $\varepsilon \in\left(0, \varepsilon_{0}\right]$.

(ii) $F_{1}$ and $F_{2}$ are $T$-periodic in $t$ ( $T$ independent of $\varepsilon$ ).

(iii) $y(t)$ belongs to $\Omega$ on the interval of time $[0,1 / \varepsilon]$.

Then the following statements hold.

(a) For $t \in[1, \varepsilon]$ we have that $x(t)-y(t)=O(\varepsilon)$, as $\varepsilon \rightarrow 0$.

(b) If $p$ is a singular point of the averaged equation (19) and

$$
\left.\operatorname{det}\left(\frac{\partial f_{1}}{\partial \mathbf{y}}\right)\right|_{\mathbf{y}=p} \neq 0
$$

then there exists a $T$-periodic solution $\varphi(t, \varepsilon)$ of equation (18) which is close to $p$ such that $\varphi(0, \varepsilon) \rightarrow p$ as $\varepsilon \rightarrow 0$.

(c) The stability or instability of the limit cycle $\varphi(t, \varepsilon)$ is given by the stability or instability of the singular point $p$ of the averaged system (19). In fact, the singular point $p$ has the stability behavior of the Poincaré map associated to the limit cycle $\varphi(t, \varepsilon)$.

In the follow we use the idea of the proof of Theorem 5(c). For more details see the section 6.3 and 11.8 of [7]. Suppose that $\varphi(t, \varepsilon)$ is a periodic solution of (18) corresponding to $y=p$ a singular point of the averaged equation (19). We linearise equation (18) in a neighbourhood of the periodic solution $\varphi(t, \varepsilon)$ and obtain a linear equation with $T$-periodic coefficients

$$
\dot{x}=\varepsilon A(T, \varepsilon) x,
$$

with $A(t, \varepsilon)=\frac{\partial}{\partial x}\left[F_{1}(t, x)+\varepsilon F_{2}(t, x, \varepsilon)\right]_{x=\varphi(t, \varepsilon)}$.

We introduce the $T$-periodic matrix

$$
B(t)=\frac{\partial F_{1}}{\partial x}(t, p) .
$$

From Theorem 5 we have $\lim _{\varepsilon \rightarrow 0} A(t, \varepsilon)=B(t)$. We shall use the matrices

$$
B_{1}=\frac{1}{T} \int_{0}^{T} B(t) d t
$$


and

$$
C(t)=\int_{0}^{t}\left[B(s)-B_{1}\right] d s
$$

Note that $B_{1}$ is the matrix of the linearized averaged equation. The matrix $C(t)$ is $T$-periodic and it has average zero. The near-identity transformation

$$
x \mapsto y=(I-\varepsilon C(t)) x
$$

writes equation (20) as

$$
\dot{y}=\varepsilon B_{1} y+\varepsilon(A(t, \varepsilon)-B(t)) y+O\left(\varepsilon^{2}\right) .
$$

We note that $A(t, \varepsilon)-B(t) \rightarrow 0$ as $\varepsilon \rightarrow 0$, and also that the characteristic exponents of equation (22) depend continuously on the small parameter $\varepsilon$. It follows that, for $\varepsilon$ sufficiently small, if the determinant of $B_{1}$ is not zero, then 0 is not an eigenvalue of the matrix $B_{1}$ and then it is not a characteristic exponent of (22). By the near-identity transformation we obtain that system (20) has not multipliers equal to 1.

\section{ACKNOWLEDGEMENTS}

The first author is partially supported by the grants MEC/FEDER MTM 2008-03437, CIRIT 2009SGR 410 and ICREA Academia. The second author is partially supported by CAPES/MECDDGU 015/2010 Brazil and Spain, process number BEX 4251/10-5.

\section{REFERENCES}

[1] R. Abraham and J.E. Marsden, Foundations of Mechanics, Benjamin, Reading, Masachusets, 1978.

[2] D. Armbruster, J. Guckenheimer, S. Kim, Chaotic dynamics in systems with square symmetry, Phys. Lett. A 140 (1989), 416-420.

[3] V.I. Arnold, V. Kozlov and A. Neishtadt, Dynamical Systems III. Mathematical Aspects of Classical and Celestial Mechanics, Third Edition, Encyclopaedia of Mathematical Science, Springer, Berlin, 2006.

[4] L. Jiménez-Lara And J. Llibre, Periodic orbits and non-Integrability of Henon-Heiles system, to appear in J. Physics A, Math. Gen.

[5] L. Jiménez-Lara And J. Llibre, Periodic orbits and non-Integrability of generalized classical Yang-Mills Hamiltonian system, Preprint, 2010.

[6] H. Poincaré, Les méthodes nouvelles de la mécanique céleste, Vol. I, Gauthier-Villars, Paris 1899.

[7] F. Verhulst, Nonlinear Differential Equations and Dynamical Systems, Universitext, Springer, 1991.

${ }^{1}$ Departament de Matemàtiques, Universitat Autònoma de Barcelona, 08193 Bellaterra, Barcelona, Catalonia, Spain

E-mail address: jllibre@mat.uab.cat

2 Departamento de Matemática, Universidade Estadual Júlio de Mesquita, 15054-000 São José do Rio Preto, Brasil

E-mail address: lroberto@ibilce.unesp.br 